\title{
TV/Series
}

$13 \mid 2018$

Diziler : les séries télévisées turques

\section{Diziler : les séries télévisées turques}

\section{Béatrice Garapon et Barbara Villez}

\section{(2) OpenEdition}

Journals

Édition électronique

URL : http://journals.openedition.org/tvseries/2320

DOI : 10.4000/tvseries.2320

ISSN : 2266-0909

\section{Éditeur}

GRIC - Groupe de recherche Identités et Cultures

\section{Référence électronique}

Béatrice Garapon et Barbara Villez, «Diziler : les séries télévisées turques », TV/Series [En ligne], 13 2018, mis en ligne le 03 juillet 2018, consulté le 14 novembre 2019. URL : http:// journals.openedition.org/tvseries/2320

Ce document a été généré automatiquement le 14 novembre 2019.

\section{(c) (i) () $\Theta$}

TV/Series est mis à disposition selon les termes de la licence Creative Commons Attribution - Pas d'Utilisation Commerciale - Pas de Modification 4.0 International. 


\title{
Diziler : les séries télévisées turques
}

\author{
Béatrice Garapon et Barbara Villez
}

1 Lorsque nous avons organisé le colloque sur les séries turques qui est à l'origine de ce numéro, en 2014 , les séries turques étaient un véritable phénomène. On ne comptait pas les articles de presse et mémoires d'étudiants qui se posaient la question d'un soft power à la turque, de la promotion du «modèle turc» par le biais de l'exportation de séries. Quelques années plus tard, en 2018, la Turquie exporte toujours autant de séries, mais il n'est plus question de « modèle démocratique » turc. Revenons d'abord brièvement sur la structuration de l'audiovisuel en Turquie, pour aborder le succès des séries.

\section{L'audiovisuel en Turquie : quelques faits sur l'âge d'or des séries turques}

2 Longtemps gardée sous le contrôle étroit de l'État, le secteur audiovisuel en Turquie a commencé à être libéralisé dans les années 1990. En 2001, une grave crise financière a nécessité un assainissement des finances publiques et plusieurs rachats de chaînes publiques par de grandes holdings. Cette situation a fait naître, en peu de temps, un grand nombre de chaînes concurrentes, et la nécessité de produire massivement du contenu, pour remplir les grilles de programmation de ces nouvelles chaînes ${ }^{1}$. Alors que, jusqu'alors, les chaînes turques se contentaient d'acheter des fictions étrangères, de programmer des jeux ou d'autres émissions de ce type, elles ont commencé à produire elles-mêmes des séries destinées à un public domestique. Ces séries (en turc dizi, au pluriel diziler), souvent très longues (rarement moins de $90 \mathrm{~min}$ ), ressemblent plus aux telenovelas sud-américaines qu'aux séries américaines. Souvent faites de romances et de drames, elles sont plutôt adressées à un public féminin ${ }^{2}$. Depuis le début des années 2000 , le secteur audiovisuel a bénéficié d'une croissance rapide, ce qui a permis d'augmenter la qualité des séries turques (qualité de l'image, acteurs, costumes, décors, sons, postproduction). La télévision reste un média très populaire en Turquie, malgré la concurrence d'internet: en 2011, un adulte passait en moyenne 3h48 par jour devant la télévision ${ }^{3}$. 
3 L'économie de l'audiovisuel en Turquie fonctionne essentiellement sur un marché de l'audience : l'État n'offre qu'un faible soutien à la production audiovisuelle, qui se finance grâce à la publicité. Ainsi, l'audiovisuel et le secteur de la publicité sont liés par une dépendance mutuelle: les publicitaires ont besoin de contenus à fort taux d'audience pour diffuser des publicités regardées par plusieurs milliers de téléspectateurs, et les chaînes ont besoin du secteur publicitaire pour se financer ${ }^{4}$. Des séries "rentables" comme Gümüş [Noor] $]^{5}$ marquaient ainsi le début en 2008-2009 d'un véritable âge d'or de la télévision turque.

4 À partir de 2008, avec le succès dans le monde arabe de la série Gümüş [Argent - en arabe Noor], ces séries se sont révélées être des produits facilement exportables. Les pays des Balkans (notamment la Grèce, la Roumanie, la Bulgarie, la Macédoine, le Monténégro, ou encore la Bosnie), le Caucase et l'Asie centrale (dont le Turkménistan, l'Azerbaïdjan, la Géorgie, le Kazakhstan ou l'Ouzbékistan), ainsi que la péninsule arabique (Qatar, Arabie saoudite) et l'Afrique du Nord (le Maroc, l'Algérie, l'Égypte), sont les principales zones d'exportation. Les raisons de ce succès sont multiples et on a beaucoup glosé sur le "modèle turc ", la diffusion de valeurs musulmanes - comme les pays d'exportation sont, pour beaucoup, des pays musulmans. En réalité, il ne faut pas perdre de vue les avantages commerciaux que présentent les séries turques par rapport aux séries américaines. Moins chères, elles sont aussi beaucoup plus longues ( 90 minutes par épisode en moyenne pour environ trente épisodes par saison) et ont l'avantage de fournir deux à quatre fois plus de contenu ${ }^{6}$.

5 Pour un pays comme la Grèce, l'offre turque présentait d'énormes avantages. À cause de sa propre situation économique, la télévision grecque produisait extrêmement peu de programmes locaux. Il était donc moins cher pour elle d'importer des séries étrangères que d'en produire, et les séries turques étaient bien plus économiques que celles émanant des États-Unis, d'un format de seulement 52 minutes. Ainsi est-elle devenue un des premiers importateurs des séries turques dans la région. Mais l'exportation des séries turques vers les Grèce a produit plusieurs effets étonnants : l'animosité envers la Turquie, installée depuis fort longtemps du fait de conflits historiques, a commencé à s'adoucir, le tourisme vers la Turquie augmente et la turcophonie gagne du terrain ${ }^{7}$. Certains personnages masculins des séries turques feraient même rêver les téléspectatrices en Grèce.

6 Néanmoins cette importante vente de séries envers la région méditerranéenne n'a pas été reçue avec autant d'enthousiasme de la part des instances politiques et religieuses d'autres pays des Balkans, de Caucase ou dans les pays arabes. Les Balkans et le Caucase et les pays de l'ancienne Union soviétique, qui craignaient une sorte d'impérialisme culturel turque et cherchaient à corriger le traitement de certains faits historiques, ont réagi par le passage de lois pour remplacer des séries turques par des programmes locaux ${ }^{8}$. L'Égypte, qui pouvait se féliciter d'une production importante des séries locales, importait néanmoins bon nombre de séries turques jusqu'au jour où un conflit politique surgit en 2013 entre Erdoğan et le gouvernement égyptien, qui a répliqué en retirant de la programmation les émissions en provenance de la Turquie. Si certains pays arabes critiquaient fortement les séries turques pour la façon dont elles représentaient des traditions religieuses, en Arabie Saoudite des leaders religieux sont allés jusqu'à demander l'interdiction de ces séries, voir menacer les propriétaires des chaînes qui les diffusaient ${ }^{9}$. Plus récemment, dans la crise entre l'Arabie saoudite et le Qatar, le gouvernement saoudien a interdit la diffusion de séries turques, afin de punir la Turquie 
pour son soutien au Qatar ${ }^{10}$. De tels conflits et interdictions continuent aujourd'hui pour des raisons religieuses, politiques et commerciales ${ }^{11}$ et montrent bien combien les séries télévisées sont devenues des enjeux à la fois commerciaux et géopolitiques majeurs pour la Turquie ${ }^{12}$.

\section{L'image de la Turquie : un soft power?}

$7 \mathrm{Si}$, entre 2009 et 2012, les séries turques ont connu un âge d'or en termes de qualité c'est en partie aussi parce que la plupart des séries iconoclastes, qui sortaient du schéma classique de la romance ou du drame, ont été produites entre ces deux dates. Après, le gouvernement turc a cherché de plus en plus activement à contrôler l'image du pays donnée par les séries et aujourd'hui, en 2018, la situation des productions est très différente.

Arrivé au pouvoir en 2001, issu d'une scission de la jeune garde du parti islamiste, l'AKP, Parti de la justice et du développement, mettait en avant un islam modéré. Ce parti était en nette rupture avec le kémalisme - du nom du fondateur de la République turque, Mustafa Kemal Atatürk, qui avait promu des réformes laïques et un modèle occidental pour la Turquie. Alors que tous les partis politiques arrivés au pouvoir en Turquie jusqu'à l'AKP se réclamaient de cette idéologie, le nouveau parti et son jeune leader, Recep Tayyip Erdoğan, faisaient figure d'outsider. Durant les années 2000, l'AKP, qui était confronté au pouvoir de l'armée dans les institutions turques, s'était donné une image de rempart de la démocratie, et, sous l'influence des négociations d'adhésion à l'Union européenne, avait engagé une série de réformes libérales. Cette libéralisation généralisée s'était ressentie dans le domaine de l'audiovisuel : plusieurs séries abordent des sujets sensibles, adoptent un ton plus libre sur le plan des mœurs, formulent une critique sociale. À partir de 2013, la censure, à travers son organe, le Conseil supérieur de l'audiovisuel (RTÜK, Radyo ve Televizyon Üst Kurulu), se fait plus sévère. Finalement, c'est avec la tentative de coup d'État du 16 juillet 2016 que tout bascule : les autorités lancent de larges purges dans tous les secteurs de l'économie, et l'audiovisuel n'est pas épargné13. Aujourd'hui, les séries turques constituent peut-être encore un secteur très profitable pour l'économie turque, mais elles restent cantonnées dans le drame et le registre comique très éloigné d'une critique de la société ou - encore moins - du pouvoir.

\section{Le cycle global television : comment comprendre et interpréter le succès des séries turques ?}

La découverte de la circulation des séries turques dans la région méditerranéenne et audelà a inspiré la création un cycle de conférences à Paris : Global Television Series ${ }^{14}$. En effet, d'après une étude d'Eurodata, la Turquie occupait la deuxième place, juste après les ÉtatsUnis, comme exportateur des séries télévisées dans le monde, suivie elle-même par la Corée $\mathrm{du} \operatorname{Sud}^{15}$. Au vu de cette importance, le premier colloque a été consacré à la télévision turque : l'industrie et les politiques des chaînes, la censure, les particularités des diziler, leur réception par les cultures étrangères, leur façon de représenter l'amour, la famille, le succès, la pauvreté, la criminalité, les institutions politiques. Les importations, les ventes de format, les festivals où les acheteurs peuvent sélectionner des séries à diffuser sur leur chaîne afin d'attirer de plus grandes audiences 
élargissait le champs d'analyse de ces fictions à des domaines divers pour étudier les récits et les images, bien sûr mais également l'industrie télévisuelle et l'économie des marchés, la réception d'audience et les représentations sociologiques véhiculées.

Le colloque qui a eu lieu à Paris en 2014 a donc réuni des universitaires spécialisés dans l'étude des séries télévisées, ainsi que des spécialistes de la Turquie, et des professionnels de la télévision turque, qui venaient témoigner directement de leur travail. Le colloque s'est organisé autour de trois axes : production, représentations et réception. Le secteur audio-visuel turque est extrêmement productif, et fondé néanmoins sur des conditions de travail extrêmement difficiles. Les acteurs et les musiciens travaillent énormément et sont très peu payés ${ }^{16}$. Les longues journées de tournage imposées sur les acteurs réduisent le coût des productions en comparaison avec d'autres régions comme les États-Unis ou l'Europe. Les directeurs de deux chaînes, une privée (Kanal D - Pelin Distas) et l'autre public (TRT- Ibrahim Eren) ont accepté d'aborder l'épineuse question de la censure et les décisions et/ou sanctions imposées par la RTÜK (organe de censure pour l'audiovisuel).

11 Le deuxième axe permettait de découvrir la façon dont les diziler représentent la société turque : bon nombre en donnent une image idéalisée. Les contributions portaient sur les séries s'adressant à la jeunesse, aux téléspectateurs conservateurs ou encore d'autres de la gauche, ce qui permettait ainsi de constater le caractère très scindé du public turc et une importante présence des séries à contenu politique malgré les mesures de censure. Mais certains thèmes sensibles comme la question kurde sont traités de manière aseptisée, pour donner une image politiquement neutre ${ }^{17}$. Certaines séries «en costumes ", notamment Le Siècle Magnifique (Muhteşem Yüzyll) offraient des fresques de l'histoire de l'Empire Ottoman. De nombreuses autres séries tournent autour du groupe familial plutôt que d'un seul personnage principal ou bien du voisinage («mahalle») où tant d'histoires s'entremêlent. Parfois, le format de certaines séries américaines est acheté pour en produire une adaptation turque. C'est le cas de Desperate Housewives; de 2011 à 2014, la version locale (Umutsuz Ev Kadınları) était diffusée un soir par semaine sur Fox TV, et la version américaine, sur une autre chaîne, un autre soir. Le téléspectateur pouvait ainsi constater les tabous de chaque société et les façons de les détourner sur le petit écran. Si par exemple le fils de Bree Van De Kamp était homosexuel, le fils de Berrin dans la version turque n'était qu'un petit malfrat, hétérosexuel mais souvent en prison. Il était trop osé, pour la série turque, d'avoir un personnage homosexuel.

12 Même si la majorité des séries étudiées ici sont des "dramas» on constate qu'il est impossible de généraliser sur les goûts et les politiques de diffusion en Turquie car beaucoup de diziler sont des comédies, traitent des questions familiales ou des romances, mais parfois les auteurs profitent de la censure, comme un outil qui stimule la créativité plutôt que d'aplatir les scénarios. Ainsi trouvent-ils des ruses pour évoquer des questions sensibles, des thèmes socio-politiques sans les nommer directement.

13 Les informations concernant le troisième axe du colloque, la réception des séries turques dans la région de la Méditerranée, étaient parmi les plus inattendues. Les réactions envers les séries turques des médias locaux des pays importateurs ont révélé les attitudes profondes envers la culture dite populaire. Par ailleurs les références, citations ou emprunts apparaissant sur les réseaux sociaux attestaient de l'impact de cette culture étrangère sur les jeunes, et les moins jeunes.

Les articles qui composent ce numéro spécial sur les séries turques ont été choisis suite au colloque. Arzu Öztürkmen, professeure au département d'histoire de l'université du 
Bosphore (Bogaziçi üniversitesi) est spécialiste de la culture télévisuelle en Turquie. Dans son article, elle retrace l'histoire de l'industrie de la télévision en Turquie, pour dévoiler la construction du dizi comme genre depuis les années 1970. L'influence des fictions étrangères, mais aussi du fonds littéraire et théâtral turc, est fondamentale pour comprendre ce genre nouveau. Elle montre que le dizi, qui touche maintenant des audiences mondiales, est un genre différent, bien que proche, des telenovelas et soap. Souvent tournées en extérieur, avec des dialogues au temps réel, les diziler sont des séries au rythme naturellement lent, dont l'univers musical et visuel est plus riche et plus diversifié que celui des telenovelas et des soap.

Les lecteurs se rendront compte grâce à l'article de Josh Carney, maître de conférences en sociologie, anthropologie et études médias à l'université américaine de Beirut, qu'en dépit des idées reçues sur le contrôle exercé sur les séries par le gouvernement, la télévision turque a bien produit des fictions politiques notamment Kurtlar vadisi [La Vallée des loups]. Il s'agit en fait d'une franchise, de plusieurs séries (des spin-off) à saisons multiples et de films qui ont connu un grand succès au point de faire l'objet de discussions dans des magazines télévisuels et des talk-shows. Josh Carney étudie La Vallée des loups (et ses dérivés) et la lecture qu'en font les téléspectateurs en Turquie à partir des théories du complot, ce qui permet de rendre compte d'un imaginaire de l'existence de conspirations politiques menaçant le pays de l'intérieur comme de l'extérieur. Cet imaginaire est confirmé par l'étude de Petra de Bruijn, enseignante à Leiden University au Pays-Bas et spécialiste du théâtre, du cinéma et de la télévision turques. Petra de Bruijn centre son article sur la chaîne Samanyolu, la chaîne privée appartenant à Fethullah Gülen, un prédicateur musulman et nationaliste, fondateur d'un mouvement destiné à renouveler et répandre la foi musulmane. Les séries diffusées et produites par cette chaîne débordent d'intrigues politiques et de signes cachés qui selon les téléspectateurs, prouvent l'existence de complots et d'actions entreprises par un deep state.

Béatrice Garapon, docteure en science politique et spécialiste de la Turquie contemporaine, a fait la découverte en 2012 d'une série qui se démarquait nettement des autres. En effet la série Kayıp şehir [La Ville perdue] était un ovni dans un paysage audiovisuel où la majorité des séries dépeignait une société moderne aux signes de richesse comme des vastes résidences, des voitures de sport et divers accessoires de luxe. Écrite par des journalistes et des écrivains, cette série novatrice modifiait considérablement l'imaginaire de la vie urbaine en Turquie. Influencée par les traditions littéraires, la culture et l'histoire moderne turques, les auteurs ont voulu donner une image réaliste, voire crue, de la société avec ses problèmes, sa violence, l'alcool, la saleté des immeubles d'un quartier pauvre, des mœurs légères de ceux qui luttent pour survivre. Rien dans Kayıp şehir n'est négligé, ni camouflé comme dans les séries dont parle Demet Luküslü où la censure est plutôt traitée comme un défi, presque un atout pour pousser la créativité des scénaristes plus loin.

Demet Luküslü maîtresse de conférence en sociologie à l'Université Yeditepe à Istanbul se concentre sur un triptyque de séries - trois feuilletons réalisés par un même homme, qui se suivent et prolongent un dialogue mis en place avec le public. À l'annulation de Leyla Ile Mecnun [Leila et le fou], la première, une suite a été produite pour continuer le discours qui critique les politiques de l'audiovisuel en Turquie aussi bien que la politique gouvernementale. L'article identifie également les sujets tabous, interdits à la télévision et les moyens que les auteurs ont trouvés pour contourner la censure. Ces séries ont ciblé directement un public de jeunes de la classe moyenne supérieure et urbaine, qui ont 
emprunté le jargon mis en place qu'ils insèrent dans leurs communications sur les réseaux sociaux. Demet Luküslü montre comment l'humour colorant ces séries a pu acquérir des significations politiques, notamment durant la mobilisation pour préserver le parc Gezi, qui a agité la Turquie en mai et juin 2013.

Jonathan Larcher clôt ce recueil d'articles avec une étude de cas sur la place de l'histoire dans la réception des séries turques sur les marchés des Balkans, notamment chez les Roumains et les Roms. L'auteur explore les contraintes locales du marché en Roumanie, où il existe une forte demande pour de grands peplums historiques. La télévision turque par l'exportation de la série Muhtesem Yüzyil [Le Siècle magnifique] comble cette demande ce qui renforce le grand succès de ce dizi. Jonathan Larcher souligne le rôle non négligeable des clips et de la musique des génériques de séries, (soit les thèmes, soit les sujets d'une chanson) qui signalent le degré de popularité d'une émission, méritant leur place comme mesure dans les études de réception.

Les chercheurs qui poursuivent les recherches sur la circulation des séries turques dévoileront certainement d'autres enjeux économiques et géopolitiques de ce commerce. Et comme le note Julien Paris, il ne faut pas négliger ces pays où les séries s'exportent car il se peut que l'un d'eux réussira un jour peut-être à modifier l'imaginaire créé par les diziler et «à imposer son récit et ses valeurs ${ }^{18}$." D'ici là, une connaissance plus approfondie de l'audiovisuel turc et son incroyable succès ces dernières années permettra d'en tirer des leçons pour les autres pays des régions de la Méditerranée.

\section{BIBLIOGRAPHIE}

ATAY, Reyhan (2018), «L'Âge d'or des séries turques dans le monde », Le Figaro, 08 février, http:// tvmag.lefigaro.fr/programme-tv/l-age-d-or-des-series-turques-dans-lemonde_4213e868-0c13-11e8-81cb-edaa50d6a916/, consulté avril 2018.

DELAHAYE, Martine (2017), « Les Séries turques ambassadrices du pouvoir », Le Monde Télévisions et Radio, 05 mai, http://abonnes.lemonde.fr/televisions-radio/article/2017/05/06/ les-series-turques-ambassadrices-du-pouvoir_5123334_1655027.html, consulté 5 mai 2018

DESPLANQUES, Erwan 2014), « Amour, gloire et Bosphore : Istanbullywood, l'usine à fictions », Télérama, 28 décembre, http://www.telerama.fr/television/amour-gloire-et-bosphoreistanbullywood-l-usine-a-fictions,120648.php, consulté 5 mai 2018.

MARILUNGO, Francesco (2016), « The City of Terrorism or a City for Breakfast. Diyarbakir's Sense of Place in the TV Series Sultan ", Middle East Journal of Culture and Communication, volume 9, issue 3, p. 275-293.

PARIS Julien (2013), « Succès et déboires des séries télévisées turques à l'international. Une influence remise en question. ", Hérodote, vol. 148, no. 1, p. 156-170.

PARIS Julien (2013), « Une production télévisuelle turque sous contraintes multiples : l'histoire, l'audience, le marché, le politique », 2 décembre, https://dipnot.hypotheses.org/608, consulté mai 2018. 
UĞUR, TANRIÖVER, Hülya (2015), « Femmes turques et séries télévisées : diversité des lectures, richesse des usages ", Genre en séries. Cinéma, télévision, médias, numéro 1, « Les séries euroméditerranéennes à l'épreuve du genre », http://genreenseries.weebly.com/ uploads/1/1/4/4/11440046/ges_n\%C2\%B01_tanri\%C3\%B6ver.pdf, p.120-146, consulté 24 avril 2018.

\section{Réseaugraphie}

https://ahvalnews2.com/neo-ottomanism/arab-tv-ban-turkish-dramas-hurts-neo-ottomandream-aw consulté 2 avril 2018

http://www.hurriyetdailynews.com/dubai-based-broadcaster-mbc-removes-turkish-showsuntil-further-notice-128252, consulté 2 avril 2018

http://www.justfocus.fr/series/series-turques-plus-vues-series-us.html. 2017 (EURODATA: http://www.mediametrie.com/eurodatatv/), mai 2018

" Turquie: l'autorité audiovisuelle autorisée à bloquer des contenus sur internet ", Huffington Post, 22 mars 2018, https://www.huffpostmaghreb.com/entry/turquie-lautoriteaudiovisuelle-autorisee-a-bloquer-des-contenus-sur-internet_mg_5ab38e09e4b008c9e5f478e8 consulté le 10 mai 2018

« Turquie : purge dans l'enseignement et l'audiovisuel », Libération, 19 juillet 2016, http:// www.liberation.fr/planete/2016/07/19/turquie-purge-dans-l-enseignement-et-1audiovisuel_1467165 consulté mai 2018.

\section{NOTES}

1. Julien Paris, «Une production télévisuelle turque sous contraintes multiples: l'histoire, l'audience, le marché, le politique ", 2 décembre 2013, https://dipnot.hypotheses.org/608, consulté mai 2018.

2. "Femmes turques et séries télévisées : diversité des lectures, richesse des usages ", Genre en séries. Cinéma, télévision, médias, numéro 1, 2015, «Les Séries euro-méditerranéennes à l'épreuve du genre », http://genreenseries.weebly.com/uploads/1/1/4/4/11440046/ges_n\%C2\%B01_tanri\% C3\%B6ver.pdf, p.120-146.

3. Paris, ibid.

4. Paris, Ibid.

5. Reyhan Atay, «L'Âge d'or des séries turques dans le monde ", Le Figaro, 08 février 2018, http:// tvmag.lefigaro.fr/programme-tv/l-age-d-or-des-series-turques-dans-le-

monde_4213e868-0c13-11e8-81cb-edaa50d6a916/. Voir aussi Martine Delahaye, «Les Séries turques ambassadrices du pouvoir ", Le Monde, 06 juin 2017, http://abonnes.lemonde.fr/ televisions-radio/article/2017/05/06/les-series-turques-ambassadrices-du-

pouvoir_5123334_1655027.html.

6. Julien Paris « Succès et déboires des séries télévisées turques à l'international. Une influence remise en question. », Hérodote, vol. 148, no. 1, 2013, p. 156-170.

7. Paris, "Succès et déboires... », op. cit,"p. 162-3.

8. Ibid., 166-7

9. Ibid., 164 .

10. https://ahvalnews2.com/neo-ottomanism/arab-tv-ban-turkish-dramas-hurts-neo-ottomandream-aw, consulté 2 avril 2018 
11. http://www.hurriyetdailynews.com/dubai-based-broadcaster-mbc-removes-turkish-showsuntil-further-notice-128252, consulté 2 avril 2018

12. https://ahvalnews2.com/neo-ottomanism/arab-tv-ban-turkish-dramas-hurts-neo-ottomandream-aw

13. «Turquie : purge dans l'enseignement et l'audiovisuel », Libération, 19 juillet 2016 http:// www.liberation.fr/planete/2016/07/19/turquie-purge-dans-l-enseignement-et-l-

audiovisuel_1467165 consulté mai 2018 ; «Turquie: l'autorité audiovisuelle autorisée à bloquer des contenus sur internet ", Huffington Post, 22 mars 2018, https://www.huffpostmaghreb.com/ entry/turquie-lautorite-audiovisuelle-autorisee-a-bloquer-des-contenus-sur-

internet_mg_5ab38e09e4b008c9e5f478e8, consultés mai 2018.

14. Les séries télévisées turques, 'diziler': production, représentations, réception en Méditerranée, La Fémis, Paris, 17-18 octobre 2014 ; Les séries d'Amérique latine, La BPI Centre Pompidou, le 14 février, 2016 ; Cap du Nord, les séries nordiques, La BPI, Centre Pompidou, le 15 janvier 2018.

15. http://www.justfocus.fr/series/series-turques-plus-vues-series-us.html. 2017 (EURODATA: http://www.mediametrie.com/eurodatatv/), consulté mai 2018.

16. Erwan Desplanques, "Amour, gloire et Bosphore: Istanbullywood, l'usine à fictions", Télérama, 28 décembre 2014, http://www.telerama.fr/television/amour-gloire-et-bosphoreistanbullywood-l-usine-a-fictions,120648.php, consulté 5 mai 2018.

17. Comme par exemple la série Sultan diffusée de mai à octobre 2012. Voir Francesco Marilungo, "The City of Terrorism or a City for Breakfast. Diyarbakir's Sense of Place in the TV Series Sultan ", Middle East Journal of Culture and Communication, volume 9, issue 3, 2016, p. 275-293.

18. Paris, «Une production télévisuelle turque... », op. cit., p. 169.

\section{RÉSUMÉS}

Issu d'un colloque tenu à Paris en octobre 2014 sur les séries turques, ce numéro revient sur le succès de ces fictions télévisées, qui a connu un pic entre 2007 et 2014. Les contributeurs de ce numéro s'interrogent sur la juste façon de comprendre et d'interpréter ce succès. L'introduction présente le paysage de l'audiovisuel en Turquie, pour expliquer ce qui a favorisé un tel essor. Elle donne ainsi des éléments concrets qui permettent d'éclairer l'exportation de ces émissions dans de nombreuses régions avoisinantes. Enfin, les auteures discutent la diffusion d'un soft power à la turque via ces séries, et montrent comment chaque article permet d'apporter une réponse, chaque fois sous un angle différent, à ces questions.

Following a conference held in Paris in October 2014 on Turkish series, this collection of articles deals with the success of these televised fictions, especially during its peak between 2007 and 2014. The articles contained here analyse the reasons for this success and offer ways to understand these programmes. This introduction presents the Turkish audiovisual landscape, in order to explain what made this triumph possible as well as the effects of these exportations to neighbouring countries. The authors discuss the diffusion of a "soft power Turkish fashion" via the series and how each article in this collection, each from a different angle, responds to the questions raised. 


\section{INDEX}

Mots-clés : Turquie, audiovisuel, séries télévisées, dizi, exportation, importation, enjeux commerciaux, enjeux géopolitiques, soft power

Keywords : Turkey, audiovisuel, TV series, dizi, export, import, commercial stakes, geopolitical stakes, soft power

\section{AUTEURS}

\section{BÉATRICE GARAPON}

Béatrice Garapon est docteure en histoire et science politique, spécialiste de la Turquie contemporaine. Elle est l'auteur d'une thèse sur les processus de démocratisation en Turquie et en particulier sur le Parti démocrate turc (1946-1960). Ancienne élève de l'École normale supérieure et diplômée de Sciences Po Paris, Béatrice Garapon a vécu plusieurs années en Turquie, en Égypte et ailleurs au Moyen-Orient.

Beatrice Garapon has a PhD in history and political science. She is a specialist of contemporary Turkey, her thesis being on the process of democratisation in Turkey particularly the success of the Democratic Party (1946-1960). She graduated from the École normale supérieure (ENS - Paris) and the Institute of Political Science in Paris. Béatrice Garapon spent several years living in Turkey, Egypt and elsewhere in the Middle East.

\section{BARBARA VILLEZ}

Barbara Villez est professeur émerite à l'Université Paris 8. De 2009 à 2017 elle a été responsable d'un réseau de chercheurs travaillant sur les séries télévisées (au LCP-IRISSO). Elle a publié de nombreux articles sur les séries judiciaires télévisées mettant en exergue la comparaison des représentations de la justice française et américaine. Son livre Séries télé: visions de la justice (PUF, 2005), traduit en anglais et réactualisé en 2009 (Television and the Legal System, Routledge) retrace plus de soixante ans de fictions judiciaires dans ces deux pays. En 2014 elle a publié Law \& Order, New York Police Judiciaire: la justice en prime time dans une collection sur les series télé aux Presses Universitaires de France.

Barbara Villez is emeritus professor at University of Paris 8. Between 2009 and 2017 she directed a network of scholars on television series at LCP-IRISSO (University Paris Dauphine and the National Research Center, CNRS). She has written numerous articles on television law series comparing televised representations of law in France and the United States. Her book Séries télé: visions de la justice (PUF, 2005), translated and updated in English (Television and the Legal System, Routledge 2009) retraces over sixty years of television courtroom dramas in these two countries. Her Law \& Order, New York Police Judiciaire: la justice en prime time was published in 2014 by Presses Universitaires de France. 